EPJ Web of Conferences 40, 06006 (2013)

DOI: $10.1051 /$ epjconf/20134006006

(C) Owned by the authors, published by EDP Sciences, 2013

\title{
Ultrafine Sm-Fe-N Particles Prepared by Planetary Ball Milling
}

\author{
G. Hadjipanayis, D. Neil and A. Gabay \\ Department of Physics and Astronomy, University of DE, Newark, DE, USA
}

\begin{abstract}
Ultrafine magnetically hard particles are needed for the bottom-up fabrication of anisotropic exchanged-coupled permanent magnets. In this study we have chosen $\mathrm{Sm}_{2} \mathrm{Fe}_{17} \mathrm{~N}_{\mathrm{x}}$ because of its high anisotropy field and large saturation magnetization. A multi-stage planetary ball milling process was used. The key is to find the right balance of energy used to mill the precursor particles; it must be high enough to break the particles and reduce their size but, not so high as to destroy the crystal structure of the nanoparticles that would lead to deterioration of the magnetic properties. First the coarse powders were subjected to a wet milling with $2.0 \mathrm{~mm}$ diameter media. In each subsequent stage the media size was reduced to maintain the milling balance. Using such a process produced particles in a size range from 100 - $800 \mathrm{~nm}$ with a coercivity as high as $10 \mathrm{kOe}$ at room temperature.
\end{abstract}

\section{Introduction}

Today there is a great deal of interest in making things smaller, lighter and more energy efficient and therefore, reduce the dependence on fossil fuels and switch to alternative energy sources. One path is to create more efficient motors and generators, the types used in today's wind turbines and electric cars. Creating super-strong exchanged-coupled nano-composite magnets would provide such an increased efficiency [1]. Although there are no size restrictions for a hard magnetic

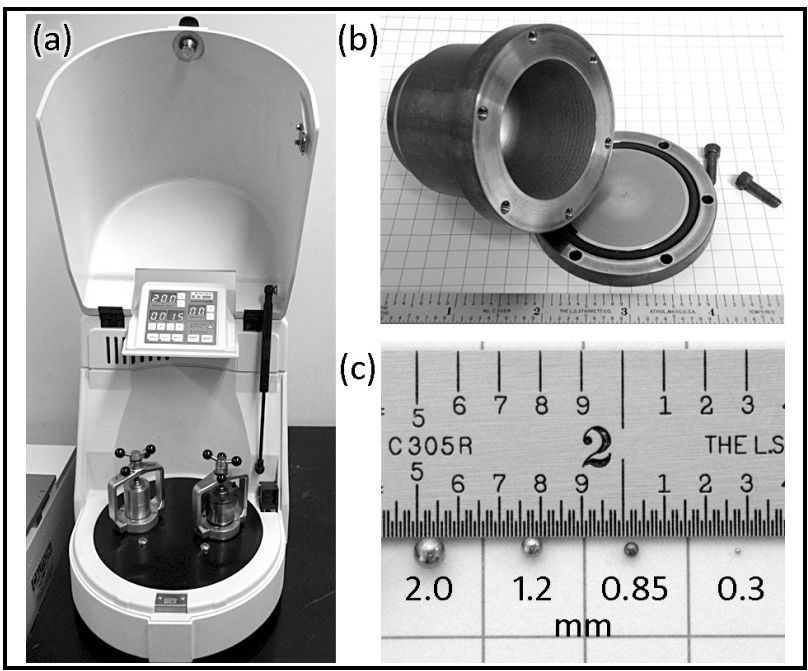

Fig. 1 (a) Planetary mill (b) sealed milling jar (c) milling

component of the exchange-coupled magnet, the size of this component ultimately determines the maximum allowed amount of the other, high-magnetization component and, therefore, the overall gain in the magnetic performance. The micron-size hard magnetic particles used in the conventional powder metallurgy of the permanent magnets and usually obtained via ball- or jet-milling can allow for only a few volume per cent of the soft magnetic phase. On the other hand, despite some moderately promising reports $[3,4,5,6]$, no technique yet emerged capable of producing large quantities of anisotropic hard magnetic nanoparticles. The material of particular interest for nanocomposite magnets is $\mathrm{Sm}_{2} \mathrm{Fe}_{17} \mathrm{~N}_{\mathrm{x}}$ with near-record saturation magnetization and very strong magnetocrystalline anisotropy [2]. In the past, many efforts were invested in preparation of polycrystalline $\mathrm{Sm}_{2} \mathrm{Fe}_{17} \mathrm{~N}_{\mathrm{x}}$ materials with randomly oriented nanograins by means of high-energy ball milling [7], melt-spinning [8] and hydrogen disproportionation desorption recombination [9]. Anisotropic single-crystal $\mathrm{Sm}_{2} \mathrm{Fe}_{17} \mathrm{~N}_{\mathrm{x}}$ particles produced via a lower-energy ball milling [10,11] are optimized for their magnetic performance (which can be excellent) and measure few microns in size. Even when mentioned, separate $\mathrm{Sm}_{2} \mathrm{Fe}_{17} \mathrm{~N}_{\mathrm{x}}$ particles of submicron size [12] are given little consideration because of their inferior properties. This paper is focused on development of viable method for preparation of submicron-sized hard magnetic particles of $\mathrm{Sm}_{2} \mathrm{Fe}_{17} \mathrm{~N}_{\mathrm{x}}$.

This is an Open Access article distributed under the terms of the Creative Commons Attribution License 2.0, which permits unrestricted use, distribution, and reproduction in any medium, provided the original work is properly cited. 


\section{Experiment}

The precursors Sm-Fe alloys were arc-melted into an ingot. Each ingot was homogenized and then crushed into a $45 \mu \mathrm{m}$ powder. Nitrogen gas at $450{ }^{\circ} \mathrm{C}$ was used to nitrogenate the powder. The powder was placed into an

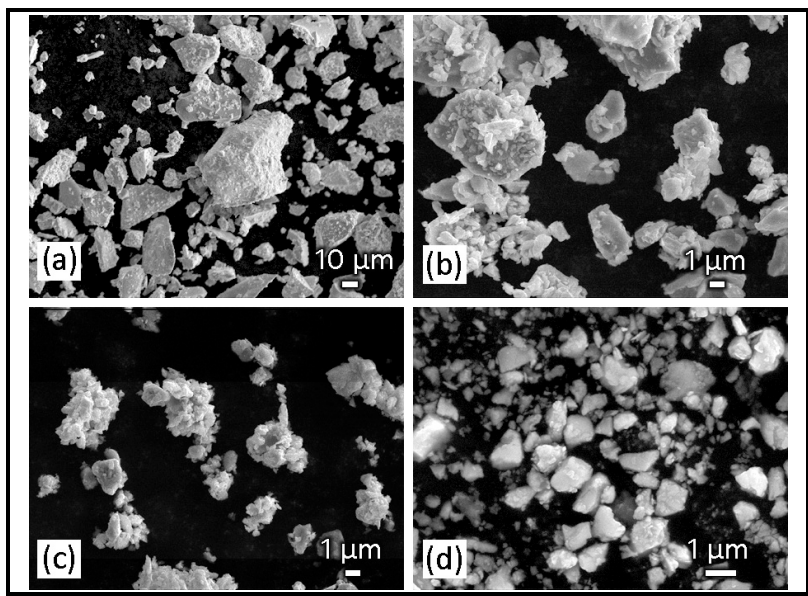

Fig. 2 (a) As made powder (b) step one $1.2 \mathrm{~mm}$ media (c) step two $0.85 \mathrm{~mm}$ media (d) step three $0.3 \mathrm{~mm}$ media

o-ring sealed high-energy ball milling (HEBM) jar with a 10:1 ball/powder ratio (Figure $1 \mathrm{~b}$ ). Ethanol was used to wet mill the material. Milling speeds from 200 to 600 rpm were used in a Fritsch Pulverisette 7 Planetary Micro Mill for these experimental runs (Figure 1a). Milling times varied from $5 \mathrm{~min}$ up to $2 \mathrm{~h}$. Milling was done in a multiple step process. In each step a smaller diameter ball was used. All milling balls are chrome steel except for the $0.3 \mathrm{~mm}$ which are made of stainless steel. The ball size was varied from $2.0 \mathrm{~mm}, 1.2 \mathrm{~mm}, 0.85 \mathrm{~mm}$ and $0.3 \mathrm{~mm}$ diameters (Figure 1c). This was done to reduce the particle size as a precursor for the subsequent step. At the end of each step the wet powder was sieved to remove the milling media and returned to the milling jar. The powder was kept wet to minimize the possibility of oxidation. Small samples of powder were dried and used for characterization. Particle morphologies and their size distributions were analyzed with a JEOL JSM-6335F SEM. Magnetic properties were measured with a Quantum Design vibrating sample magnetometer on field-aligned powder samples embedded into wax.

\section{Results and Discussion}

Many milling variations were tried during this experimentation. Single, two and three step ball milling trials were run; runs at low energy for long durations and runs at high energy for short durations. Different combinations of milling media, including mixed ball size batches. Mixed media size batches tended to over mill the material in that the larger media continued to apply higher energy than was necessary after the larger particles had been reduced. It was also found that if to small a

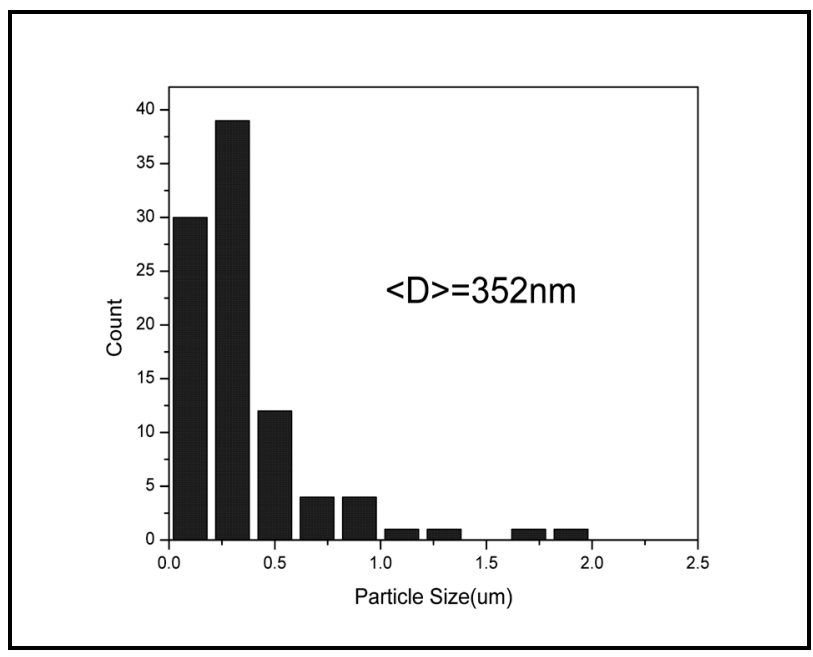

Fig. 3 Three step milling run particle size distribution

media was used to start the process, the larger particles would not be reduced before the smaller ones had been over milled. The best particles produced so far are from a 3 step run that had a slurry mean size of $352 \mathrm{~nm}$ (Figure 2\&3). A remanence $M_{\mathrm{r}}$ of $90.7 \mathrm{emu} / \mathrm{g}$ with an intrinsic

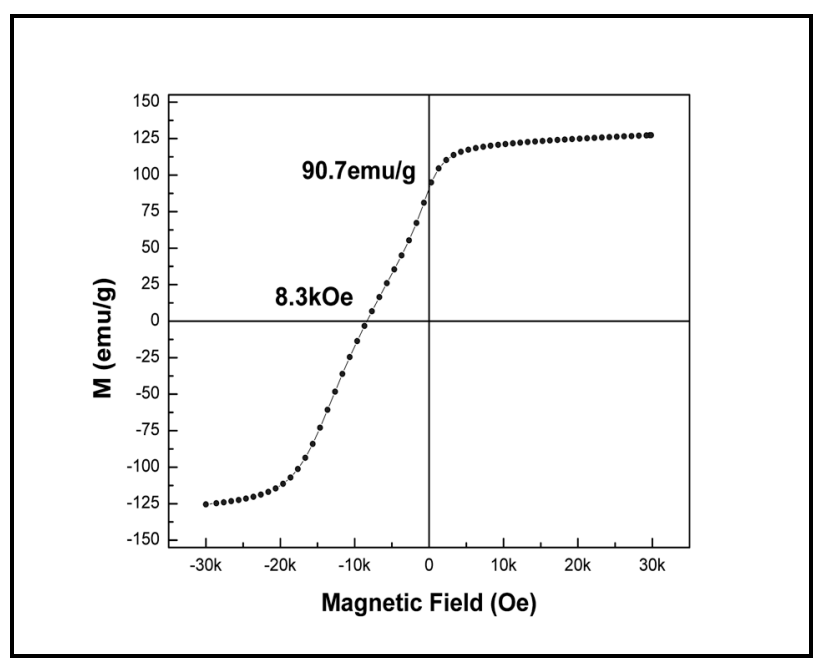

Fig. 4 Uncorrected room temperature VSM data from a three step run

coercivity $H_{\mathrm{c}}$ of $8.3 \mathrm{kOe}$ (Figure 4 ). The three step process has yielded slightly smaller particles but, the magnetic properties where poor. A limited number of runs have been made using the 3 step process with the stainless steel $0.3 \mathrm{~mm}$ diameter balls. This process needs to be optimized to determine if nanoparticles (size 
smaller than $100 \mathrm{~nm}$ ) can be also produced. Additional steps may need to be added to maintain the proper milling energy. The stainless steel $0.3 \mathrm{~mm}$ balls may be too soft and to large to effectively mill the harder materials to the desired size of under $100 \mathrm{~nm}$. Alternative harder and smaller milling media are being investigated.

\section{Conclusions}

The planetary milling process has allowed us to produce 4 to 6 gram quantities of particles smaller than $500 \mathrm{~nm}$ with good magnetic properties. With smaller, harder media it may be possible to decrease the particle size even further and obtain nanoparticles, which are best suited for nanocomposite magnets.

\section{Acknowledgement}

This work is supported by ARPA-E program.

\section{References}

1. D.J. Sellmyer, Nature 420, 374 (2002).

2. R. Skomski and J.M.D. Coey, Phys. Rev. B 48,15812 (1993).

3. Y. Wang, Y. Li, C. Rong, and J.P. Liu, Nanotechnology 18, 465701 (2007)

4. V.M. Chakka, B. Altuncevahir, Z.Q. Jin, Y. Li, and J.P. Liu, J. Appl. Phys. 99, 08E912 (2006)

5. N.G. Akdogan, G.C. Hadjipanayis, and D.J. Sellmyer, J. Appl. Phys. 105, 07A710 (2009)

6. B.Z. Cui, L.Y. Zheng, D. Waryoba, M. Marinescu, and G.C. Hadjipanayis, J. Appl. Phys. 109, 07A728 (2011)

7. K. Schnitzke, L. Schultz, J. Wecher, M. Katter, Appl. Phys. Lett. 57, 2853 (1990).

8. F.E. Pinkerton, C.D. Fuerst, Appl. Phys. Lett. 60, 2558 (1992).

9. H. Nakamura, S. Sugimoto, M. Okada, M. Homma, Mater. Chem. Phys. 32, 280 (1992).

10. H. Izumi, K. Machida, A. Shiomi, M. Iguchi, K. Noguchi, G. Adachi, Chem. Mater. 9, 2759 (1997).

11. A. Kawamoto, T. Ishikawa, S. Yasuda, K. Takeya, K. Ishizaka, T. Iseki, K. Ohmori, IEEE Trans. Magn. 35, 3322 (1999).

12. R. Arlot, H. Izumi, K. Machida, D. Fruchart, G. Adachi, J. Magn. Magn. Mater. 172, 119 (1997). 